\title{
Breast Reconstruction with Silicone Prosthesis Immediately Following Polyacrylamide Gel Removal
}

\author{
Bingjing Leng, Jie Ren, Zhixue Yang, Jinrong Wei and Guoqin Jiang \\ Department of Breast and Thyroid Surgery, The Second Affiliated Hospital of Soochow University, Suzhou, China
}

\begin{abstract}
Objective: To determine the feasibility and efficacy of breast reconstruction with silicone prosthesis immediately following polyacrylamide gel removal.

Study Design: An observational study.

Place and Duration of Study: Department of Breast and Thyroid Surgery, the Second Affiliated Hospital of Soochow University, Suzhou, China, from November 2013 to May 2017.

Methodology: Twenty-seven patients with serious complications resulting from polyacrylamide gel (PAAG) injection were operated in our hospital. Using an inframammary fold incision, the PAAG and surrounding tissues were completely removed. All patients were received mastopexy and immediate breast reconstruction.

Results: Of all patients, 26 reported relief of preoperative complications, without obvious postoperative complications. Only one patient developed recurring infections after surgery and removed the prostheses.

Conclusion: After removal of PAAG, mastopexy followed by immediate breast reconstruction allows the patient to obtain a satisfactory appearance without serious complications. The key to successful breast reconstruction with prostheses first lies with the complete excision of PAAG and surrounding tissues.
\end{abstract}

Key Words: PAAG, Injection breast augmentation, Prosthesis, Breast reconstruction, Mastopexy.

How to cite this article: Leng B, Ren J, Yang Z, Wei J, Jiang G. Breast reconstruction with silicone prosthesis immediately following polyacrylamide gel removal. J Coll Physicians Surg Pak 2019; 29(11):1092-5.

\section{INTRODUCTION}

Polyacrylamide gel (PAAG) is a transparent, gelatinous material that was once considered to be a safe and stable material for soft tissue filling, and was widely used in the cosmetic surgery. ${ }^{1}$ In 1987, PAAG was first used for the filling of breast, facial and other parts. From 1993 to 1995, PAAG was approved for clinical use in Ukraine, Russia, Italy, and France. By 1997, PAAG was used in China, primarily for injection breast augmentation. Then in 1999, PAAG was approved for listing in China. Within a few years, thousands of women had received PAAG injection for breast augmentation, and many of these were administered in unqualified hospitals. ${ }^{2}$ However, many women received PAAG injections for breast augmentation began to present with a variety of complications. ${ }^{2-5}$ Between 2002 and 2005, the National Drug Adverse Drug Reaction Center received 183 reports of adverse event monitoring reports related to PAAG, of which 161 were for breast augmentation. On April 30, 2006, the State Food and Drug Administration of China completely banned the production, sale, and use of PAAG.

Correspondence to: Guoqin Jiang, Department of Breast and Thyroid Surgery, The Second Affiliated Hospital of Soochow

University, No. 1055, San-Xiang Road, 215004, Suzhou, China E-mail: jiang_guoqin@hotmail.com

Received: January 08, 2019; Revised: April 25, 2019;

Accepted: June 14, 2019
Silicone prostheses were first invented by Thomas Cronin and Frank Gerow in collaboration with Dow Corning Company in 1961. Silicone breast prostheses have been established as a safe and stable material for breast augmentation through continuous research and improvement. Although prosthesis displacement, capsule contracture, and other local complications may occur, 6,7 silicone prostheses can be removed easily and replaced to remedy these issues. At the end of 2006, the U.S. Food and Drug Administration (FDA) approved the production of silicone prostheses by Allergan Company and Mentor Company. 6

The purpose of this study was to determine the feasibility and efficacy of immediate breast reconstruction with silicone prosthesis in breast augmentation patients following removal of PAAG.

\section{METHODOLOGY}

Between November 2013 and May 2017, 27 patients aged 33 to 56 years underwent removal of PAAG injections at the Second Affiliated Hospital of Soochow University. Patients had initially received PAAG injections 11-18 years prior age with an injection volume of $80-200 \mathrm{ml}$ per side (average $144 \mathrm{ml}$ per side). Twentyseven cases $(100 \%)$ had obvious mass or induration, 18 cases $(66.7 \%)$ had altered shape or asymmetry, including malformation, 14 cases $(65.8 \%)$ had pain, and two cases $(7.4 \%)$ of the injection shifting. 
Patients with serious complications after PAAG injection were selected for treatment. These patients had never received an injection removal operation and, had no infection before surgery. All patients signed informed consent for this study. This study has been approved by the Ethics Committee of the Second Affiliated Hospital of Soochow University.

Breast MRI and ultrasound were performed prior to the operation to observe the distribution of PAAG, determine whether a complete capsule had formed, estimate volume and degree of infiltration, and diagnose any malignant lesions. MRI results were also used to assess the suitability of implanting a silicone prosthesis, based on residual gland size as well as skin thickness and integrity (Figure 1).

Surgery was performed under general anesthesia in the beach chair position (semi-reclining position, Figure 2a). This positioning allows the breasts in a natural position, which provides optimal observation of breast size and shape during surgery. The nipple-areola complex, sternal notch and inframammary fold (IMF) were used as anatomical markers. To determine the position for new nipples, a preoperative measurement of each breast was completed according to Chinese women's normal body surface distribution data, and the new inframammary fold was positioned $5.5 \mathrm{~cm}$ away from the new areola (Figure 2a). In general, the sternal notch to the nipple and the mid-clavicle to the nipple each measured $19-21 \mathrm{~cm}$, whereas the nipple to the IMF measured $5-7 \mathrm{~cm} \cdot{ }^{8-10}$ The symmetry was ensured.

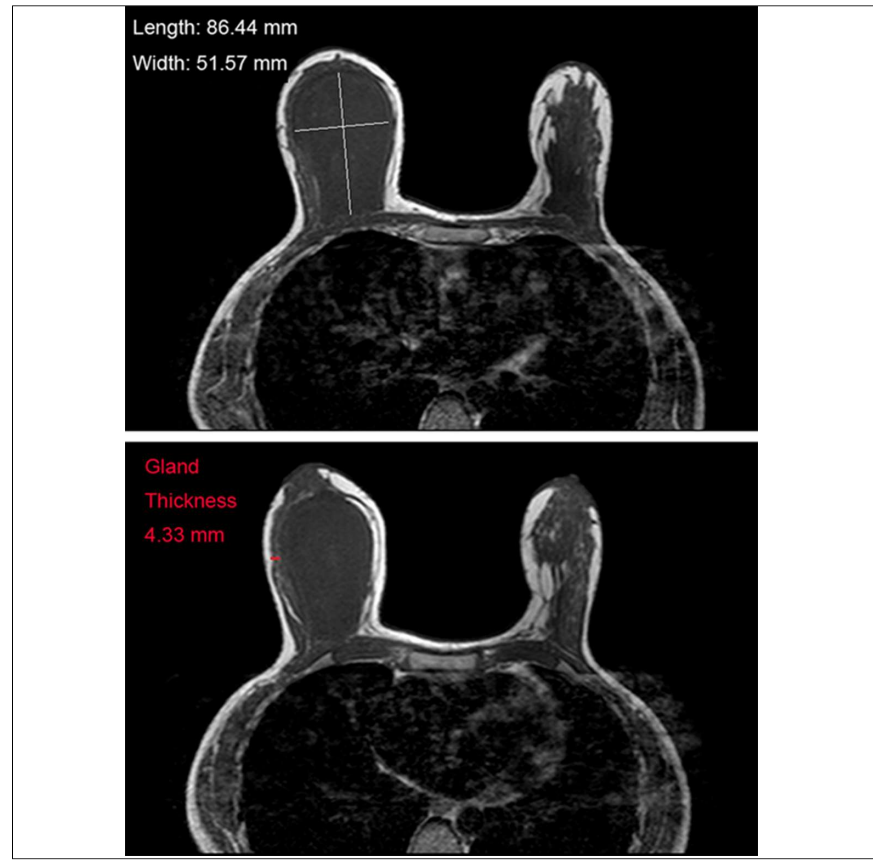

Figure 1: Bilateral breast MRI images. A patient with the bilateral posterior mammary space almost entirely composed of PAAG and completely enclosed by a capsule, with a length of $86.44 \mathrm{~mm}$ and a width of $51.57 \mathrm{~mm}$. The mammary gland is only $4.33 \mathrm{~mm}$ thick, but remains intact. There are no defects of local glands or skin, no air bubbles in the breast injection, and no local liquefaction performance, therefore this case is suitable for immediate prosthetic breast reconstruction.
An incision was made $5-7 \mathrm{~cm}$ from the IMF, to reveal the glands and submammary space. The fibrous capsule that formed around the gel was located and carefully removed in its entirety. Induration and deactivated organisation around the capsule was removed completely. The normal organisation was kept intact as much as possible. The residual cavity was rinsed with a large amount of povidone-iodine to prevent infection and capsule contracture. ${ }^{11}$

After PAAG removal, mastopexy was performed through dual-circle circumareolar and inverted $\mathrm{T}$ incisions (Figure 2b). Skin excision was determined by the degree of skin laxity, and was either concentric or biased to reposition the nipple, with nipple symmetry maintained during the operation. Then, the defect degree of residual glands was checked and repaired by the defective line. Implants for breast reconstruction were placed under the glands. The space in which the implants were placed was without defects to ensure no direct contact with adipose tissue or skin. ${ }^{12}$

Prosthesis size was measured using a dilator. The nipple and areola were located using the method described

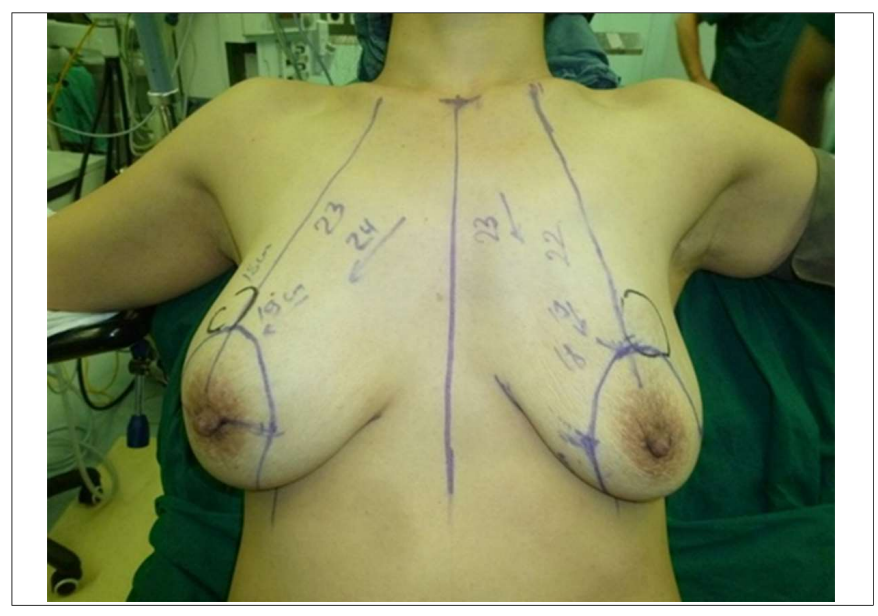

Figure 2a: The beach chair position and a preoperative measurement to determine the position of new nipples and inferolateral fold, determined according to the Chinese women's normal body data.

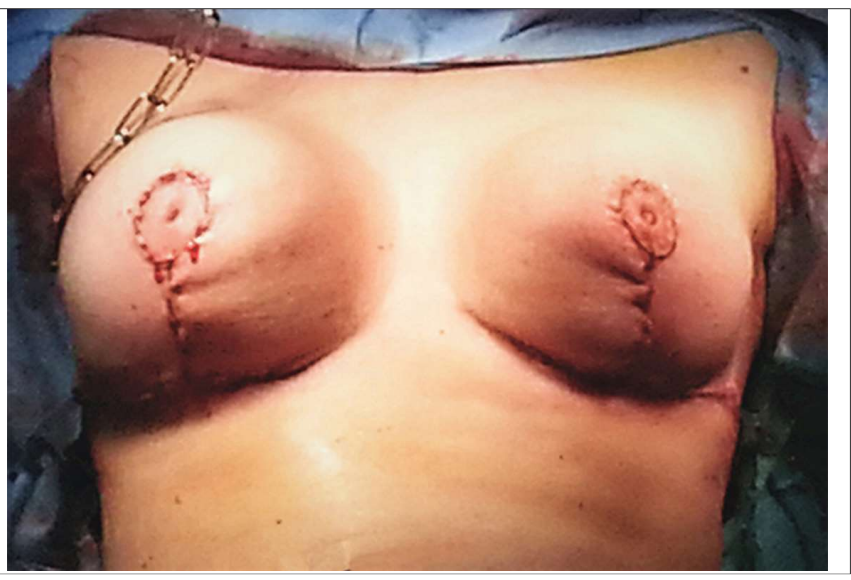

Figure 2b: Prosthesis appearance after implantation. Mastopexy, a breast lift, raises and firms the breasts by removing excess skin and tightening the surrounding tissue to reshape and support the contour of the new breast. 


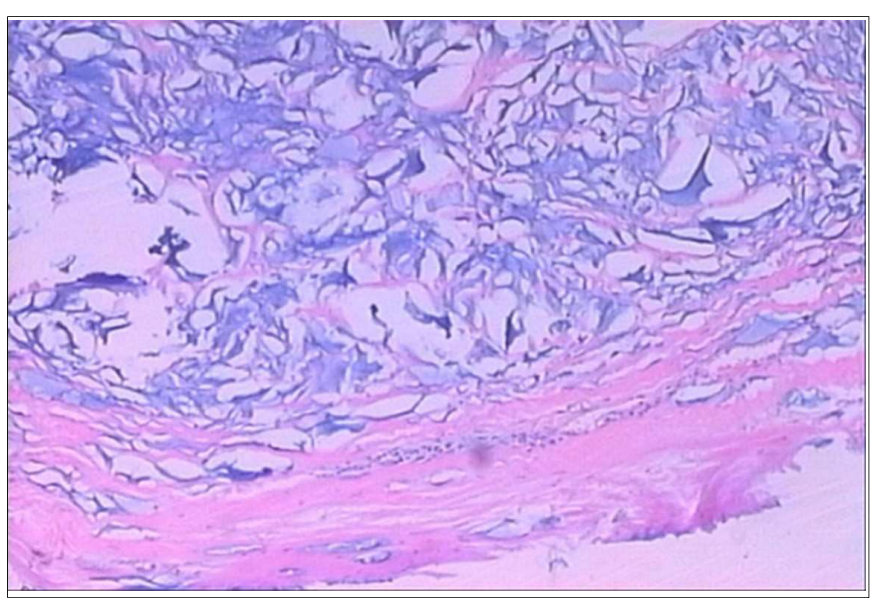

Figure 3: Pathological examination of all intraoperative excisions showed a large number of non-structural foreign bodies and proliferative fibrous tissue in the gland, including multinucleated giant cells and foreign body granulomas.

previously, the IMF was fixed to the chest wall, the expander was placed in the submammary space, and then normal saline (NS) was injected to create a satisfactory breast shape. The size of the prosthesis was dependent upon the amount of NS injected. The prosthesis was lubricated with diluted povidone-iodine, placed in the submammary space (Figure $2 b$ ).

All patients were given cephalosporin 30 minutes before and for 3 days following surgery to prevent infection. Additionally, all surgical instruments and gloves were changed after removing the PAAG injection. After surgery, patients wore elastic chest or plastic bras for at least 1 month to prevent prosthesis slippage.

\section{RESULTS}

Of all patients, 26 (average 42.51 years) reported relief of preoperative complications, without obvious postoperative complications. Only one patient developed an infection and subcutaneous hydrops at the left breast, a month after surgery. Bacterial culture of hydrops was positive for Pseudomonas aeruginosa, which was improved after treatment with cefoperazone sulbactam. However, the patient had subcutaneous hydrops in the left breast twice in a month, requiring removal of the left silicone prosthesis. All patients' follow-ups were completed for two years after the operation, and no severe complications occurred in any patient.

Postoperative pathological examination of all tissues showed a large number of non-structural foreign bodies and proliferative fibrous tissue in the gland, including multinucleated giant cells and foreign body granulomas (Figure 3).

\section{DISCUSSION}

The purpose of this study was to determine the feasibility and effectiveness of immediate breast reconstruction with silicone prostheses in women with enlarged breasts after removal of PAAG.
There are two primary ways to remove PAAG, and the authors believe that the open approach is more advantageous than aspiration. PAAG diffuses and moves between tissues due to gravity and muscle extrusion forces, resulting in hydrogel deformation and displacement. ${ }^{5}$ The correct site to inject PAAG is in the loose tissue behind the gland, but this site can be difficult to accurately locate. We found PAAG that was improperly injected into the gland, pectoralis major and minor muscles, and even behind the muscle, which maked complete removal difficult. The aspiration method was performed under local anesthesia, identified the location of PAAG cyst with ultrasound guidance, then repeatedly rinsed and aspirated. 4 However, the PAAG and its capsule could not be completely removed using the blinded suction technique, resulting in PAAG malposition and worsening of tissue denaturation. ${ }^{13,14}$ Compared to aspiration, the open approach with IMF incision ${ }^{13}$ provides better visualization, and when combined with preoperative imaging, allows for more accurate location of PAAG lesions in order to avoid unnecessary damage to normal glands. Moreover, the open approach allows for complete removal of the PAAG, the capsule, and the inactivated tissue, which reduces the risk of postoperative local infection. More importantly, the open approach allows for immediate breast reconstruction with silicone implants.

In order to yield the best results, we used anatomical textured surface silicone prostheses. A large number of studies have shown that a textured surface prosthesis can significantly reduce the incidence of postoperative capsule contracture and maintain long-term stability of prosthesis morphology and position. ${ }^{15}$ PAAG injections were distributed in the lower pole of the breast due to gravity while the glands had moved upward, and therefore, upon removal of the injection, more glands remained in the upper pole of the breast than the lower. Accordingly, the lower part of the anatomical prosthesis was fuller, suited to the shape of the breast after PAAG removal, allowing maintenance of normal breast shape.

For patients with slight tissue damage that places strong value on appearance, immediate breast reconstruction with prosthesis implantation is appropriate. Immediate reconstruction is also easier to perform, technically, because there is typically less scarring and contracture than with delayed reconstruction. Immediate reconstruction also has important psychological benefits including decreased anxiety and improved self-image. ${ }^{16}$

Although prosthesis implantation has the above benefits, its common complications include capsule contracture, prosthesis slippage, prosthetic rupture, infection, skin necrosis, etc. Capsular contracture is the most significant long-term risk with implant reconstructions. ${ }^{17}$ The authors used textured surface silicone prostheses and povidone-iodine to flush the residual cavity intraoperatively to prevent capsular contracture. With respect to infection, some reports have shown large 
microvascular formation 2-4 weeks after PAAG injection, and the microvasculature in the capsule degraded with time, but if subjected to external forces, would easily rupture and cause a local inflammatory reaction. Furthermore, the pressure from PAAG on the glands caused impaired local blood circulation, resulting in reduced tissue healing ability. Together, these effects change the compliance of surrounding tissues, making them easier to damage, resulting in aseptic inflammation. Moreover, low-grade bacterial infections may often occur with long-term prosthetic implants. Bacteria are capable of surviving for extended periods of time, especially if they are protected from host defenses within a proteinaceous biofilm of fibrinogen and fibronectin, 18,19 which can ultimately lead to the development of antibiotic resistance. ${ }^{18,20}$ Because of the shorter duration and low occurrence of such infections, further analysis is needed in future clinical work to obtain more detailed clinical documentation and data.

\section{CONCLUSION}

After PAAG removal, mastopexy followed by immediate breast reconstruction allows the patient to obtain a satisfactory appearance without causing serious complications. The key to successful breast recons-truction with prosthesis first lies with complete excision of PAAG and its surrounding tissues; and our research is focused on identifying the best method to achieve this.

\section{ETHICAL APPROVAL:}

This study has been approved by the Ethics Committee of the Second Affiliated Hospital of Soochow University.

\section{PATIENTS' CONSENT:}

All patients signed informed consent for this study.

\section{CONFLICT OF INTEREST:}

Authors declared no conflict of interest.

\section{AUTHORS' CONTRIBUTION:}

$\mathrm{BL}$, JR: Substantial contributions to the conception or design of the work and drafting the work or revising it critically for important intellectual content; final approval of the version to be published; agreement to be accountable for all aspects of the work.

ZY, JW: Revised it critically for important intellectual content; final approval of the version to be published.

GJ: Final approval of the version to be published; agreement to be accountable for all aspects of the work.

\section{REFERENCES}

1. Christensen LH, Breiting VB, Aasted A, Jørgensen A, Kebuladze I. Long-term effects of polyacrylamide hydrogel on human breast tissue. Plast Reconstr Surg 2003; 111:1883-90.

2. Cheng NX, Wang YL, Wang JH, Zhang XM, Zhong $\mathrm{H}$. Complications of breast augmentation with injected hydrophilic polyacrylamide gel. Aesthetic Plast Surg 2002; 26:375-82.
3. Qiao Q, Wang X, Sun J, Zhao R, Liu Z, Wang Y, et al. Management for postoperative complications of breast augmentation by injected polyacrylamide hydrogel. Aesthetic Plast Surg 2005; 29:156-61.

4. Wu J, Zhang X, Zhao Q, Mao D, Lu X. Vacuum sealing drainage in the treatment of migrated polyacrylamide hydrogel after breast augmentation: a case report. Breast Care (Basel) 2014; 9:273-5.

5. Patlazhan G, Unukovych D, Pshenisnov K. Breast reconstruction and treatment algorithm for patients with complications after polyacrylamide gel injections: A 10-year experience. Aesthetic Plast Surg 2013; 37:312-20.

6. Winkler E, Regev E, Bar-Meir E, Orenstein A. Silicone gel-filled breast implants and breast cancer: An update and safety. Harefuah 2004; 143:222-6, 244.

7. Tindholdt TT, Mesic H, Tønseth KA, Harbo SO. 40 years of silicone breast implants. Tidsskr Nor Laegeforen 2005; 125:739-41.

8. Liu YJ, Thomson JG. Ideal anthropomorphic values of the female breast: Correlation of pluralistic aesthetic evaluations with objective measurements. Ann Plast Surg 2011; 67:7-11.

9. Tepper OM, Unger JG, Small KH, Feldman D, Kumar N, Choi M, et al. Mammometrics: The standardization of aesthetic and reconstructive breast surgery. Plast Reconstr Surg 2010; 125: 393-400.

10. Penn J. Breast reduction. Br J Plast Surg 1955; 7:357-71.

11. Gaucher S, Maladry D, Martin A, Benachour I, Philippe HJ, Grabar S. Efficacy and safety of povidone-iodine irrigation in reducing the risk of capsular contracture in aesthetic breast augmentation: A systematic review and meta-analysis. Plast Reconstr Surg 2016; 138:368e-9e.

12. Cordeiro PG, McCarthy CM. A single surgeon's 12-year experience with tissue expander/implant breast reconstruction: Part II. An analysis of long-term complications, aesthetic outcomes, and patient satisfaction. Plast Reconstr Surg 2006; 118:832-9.

13. Mu DL, Luan J, Mu LH, Wang LY, Liu C, Wang KM, et al. Repeated operation for removement of polyacrylamide hydrogel from breast. Zhonghua Zheng Xing Wai Ke Za Zhi 2008; 24:187-9.

14. Yu L, Wang J, Zhang B, Zheng DN, Zhu C. Treatment of breast injection with polyacrylamide hydrogel with infiltrated fascia capsule removal: Report on 104 cases. Aesthetic Plast Surg 2012; 36:1120-7.

15. Barnsley GP, Sigurdson LJ, Barnsley SE. Textured surface breast implants in the prevention of capsular contracture among breast augmentation patients: A meta-analysis of randomized controlled trials. Plast Reconstr Surg 2006; 117: 2182-90.

16. Fernández-Delgado J, López-Pedraza MJ, Blasco JA, Andradas-Aragones E, Sánchez-Méndez JI, Sordo-Miralles G, et al. Satisfaction with and psychological impact of immediate and deferred breast reconstruction. Ann Oncol 2008; 19:1430-4.

17. Vandeput JJ, Nelissen M. Considerations on anthropometric measurements of the female breast. Aesthetic Plast Surg 2002; 26:348-55.

18. Tang L, Eaton JW. Inflammatory responses to biomaterials. Am J Clin Pathol 1995; 103:466-71.

19. Kao WJ, Lee D. In vivo modulation of host response and macrophage behavior by polymer networks grafted with fibronectin-derived biomimetic oligopeptides: The role of RGD and PHSRN domains. Biomaterials 2001; 22:2901-9.

20. Edwards R, Harding KG. Bacteria and wound healing. Curr Opin Infect Dis 2004; 17:91-6. 